OPEN ACCESS

Edited by:

Sreekanth Kumar Mallineni,

Majmaah University, Saudi Arabia

Reviewed by:

Rosa Helena Wanderley Lacerda, Federal University of Paraíba, Brazil

Yuan Liu,

University of Pennsylvania,

United States

Sirma Angelova,

Medical University of Varna, Bulgaria

*Correspondence:

Thaís Manzano Parisotto

thais.parisotto@usf.edu.br

Specialty section:

This article was submitted to

Pediatric Dentistry,

a section of the journal

Frontiers in Dental Medicine

Received: 31 May 2021

Accepted: 22 July 2021

Published: 17 September 2021

Citation:

Sabella FM, Feiria SNB, Ribeiro $A A$,

Theodoro LH, Höfling JF, Parisotto TM and Duque $C$ (2021) Exploring the

Interplay Between Oral Diseases, Microbiome, and Chronic Diseases

Driven by Metabolic Dysfunction in

Childhood.

Front. Dent. Med. 2:718441.

doi: 10.3389/fdmed.2021.718441

\section{Exploring the Interplay Between Oral Diseases, Microbiome, and Chronic Diseases Driven by Metabolic Dysfunction in Childhood}

\author{
Fernanda Maria Sabella ${ }^{1}$, Simone Nataly Busato de Feiria ${ }^{2}$, Apoena de Aguiar Ribeiro ${ }^{3}$, \\ Letícia Helena Theodoro ${ }^{4}$, José Francisco Höfling ${ }^{2}$, Thaís Manzano Parisotto ${ }^{1 *}$ and \\ Cristiane Duque ${ }^{5}$ \\ ${ }^{1}$ Laboratory of Clinical and Molecular Microbiology, São Francisco University - USF, Bragança Paulista, Brazil, ${ }^{2}$ Department \\ of Oral Diagnosis, State University of Campinas - UNICAMP, Piracicaba Dental School, Piracicaba, Brazil, ${ }^{3}$ Division of \\ Diagnostic Sciences, University of North Carolina at Chapel Hill-Adams School of Dentistry, Chapel Hill, NC, United States, \\ ${ }^{4}$ Department of Diagnostic and Surgery, Periodontics Division, São Paulo State University - UNESP, Araçatuba School of \\ Dentistry, Araçatuba, Brazil, ${ }^{5}$ Department of Preventive and Restorative Dentistry, São Paulo State University - UNESP - \\ Araçatuba School of Dentistry, Araçatuba, Brazil
}

Oral childhood diseases, such as caries and gingivitis, have much more than a local impact on the dentition and tooth surrounding tissues, which can affect systemic conditions. While the mouth is frequently exposed to microbial stressors that can contribute to an inflammatory state in the entire body, chronic disorders can also interfere with oral health. Sharing common risk factors, a dynamic interplay can be driven between 1. dental caries, gingivitis, and type I diabetes mellitus, 2. early childhood caries and obesity, and 3. caries and cardiovascular diseases. Considering that there are $\sim 2.2$ billion children worldwide and that childhood provides unique opportunities for intervention targeting future health promotion, this review is of prime importance and aimed to explore the relationship between the oral microbiome and oral chronic diseases driven by metabolic dysfunction in childhood.

Keywords: mouth, bacteria, obesity, cardiovascular disease, type I diabetes

\section{INTRODUCTION}

The mouth is a part of the human body and cannot be considered independent. The oral cavity harbors a diverse microbiome and the second largest number of microorganisms after the gut (1), with $\sim 500-700$ species (2). There are many distinct niches in the oral cavity that characterize a complex habitat providing shedding (soft tissues/mucosa) and non-shedding (teeth) surfaces for microbial colonization (1). The dysbiotic state of the oral microbiome triggers the most common biofilm-mediated oral diseases in children: caries and gingivitis (2).

Dental caries affects more than 530 million children worldwide (3) and is characterized by tooth demineralization due to the action of organic acids after bacterial dietary substrate fermentation $(4,5)$, while gingivitis is characterized by bleeding and swelling due to the initial inflammatory process of the gums, which can progress to the destruction of tooth-supported tissues (periodontitis) (6). Both diseases culminate in tooth loss, prejudicing the mastication process, phonetics, respiration, swallowing, and even the quality of life. 
Advances in the knowledge of how host-associated microbial communities promote or protect against pathogenic microbes and how microorganisms contribute to inflammatory diseases are extremely important. In light of this, studies targeting the oral microbiota in health and disease will provide valuable information on the functional and metabolic changes in diverse pathological states, as well as the identification of molecular signatures, which could lead to assertive therapies considering precision medicine (1). Interestingly, oral samples are easy to collect, and, therefore, studies in this regard have been increasing in the past few years. Progress in the field of molecular biology has led to culture-independent techniques, which have revealed many uncultivable microorganisms that better represent the oral microbiota and its complexities.

Systemic diseases such as obesity, cardiovascular problems, and type I diabetes mellitus (T1DM) have been shown to be influenced by dental plaque-associated oral diseases. It should be noted that oral bacteria are frequently swallowed along with saliva and solid and liquid foods during the digestion process, reaching the stomach and gut (7). Moreover, studies have demonstrated that gut microbial communities are associated with obesity (8-11) and are associated with T1DM through the immune system (12). Immunological changes in the gut can be reflected in the pancreas, where insulin is produced in response to increasing glucose levels in the bloodstream (12). In addition, as tooth nourishment is derived from the pulp and blood vessels from the surrounding tissues, oral bacteria can also spread into many organs, such as the heart via the bloodstream (13).

In light of the above knowledge, oral diseases have much more than a local impact on the dentition and tooth contiguous tissues, interacting with systemic conditions. However, chronic disorders can also interfere with oral health. A dynamic interplay can be driven between 1. dental caries, gingivitis, and T1DM; 2. early childhood caries and obesity, and 3. caries and cardiovascular diseases as they share common risk factors (Figure 1). Thus, the present review is an attempt to investigate the relationship between the oral microbiome and oral and chronic diseases driven by metabolic dysfunction in childhood.

\section{SUBSECTIONS AND DISCUSSION}

\section{T1DM}

Diabetes mellitus is a group of chronic metabolic diseases characterized by elevated levels of blood glucose as a result of defects in insulin production, action, or both (14). The most common type of diabetes mellitus in children and adolescents is type 1 or insulin-dependent diabetes (juvenile or childhood-onset diabetes). T1DM is caused by genetic autoimmune destruction of $\beta$-cells in the pancreas, in which all or a subset of islets in the pancreas lack insulin-secreting $\beta$-cells, leading to hyperglycemia and a decrease in insulin production (15). The production of multiple islet autoantibodies can be precipitated by several environmental factors, including enterovirus infections, nutritional factors (deficiency of vitamin $\mathrm{D}$, excessive consumption of cow milk proteins and nitrates) excessive amounts of glucagon, epinephrine, growth hormones, glucocorticoids, and thiazides and others (16-18). According to
$\mathrm{WHO}$, there are large differences in the incidence and prevalence of T1DM, ranging from over 60 to under 0.5 cases annually per 100,000 children aged under 15 years (19). The clinical symptoms of T1DM are polydipsia, polyphagia, polyuria, weight loss, blurred vision, difficulty concentrating, hypotension, abdominal pain, and dehydration, among others. Laboratory findings are hyperglycemia, glycosuria, and ketonuria (20).

\section{DENTAL CARIES, GINGIVITIS, AND T1DM Caries and T1DM}

The oral cavity is composed of several ecosystems, such as teeth, gingival tissues, tongue, mucosa, palate, and tonsils that harbor diverse bacteria, fungi, or viruses that coexist in symbiosis to maintain a healthy state. When a disturbance in the diversity and proportions of species or taxa within the microbiota occurs (dysbiosis), disease-promoting microorganisms proliferate, causing pathologies such as dental caries, gingivitis, and periodontitis (21). The microbiota of the oral cavity can also play role in many systemic diseases such as diabetes, cardiovascular diseases, and obesity (22).

Dental caries is a biofilm-mediated, diet modulated, multifactorial, non-communicable, dynamic disease resulting in enamel demineralization, determined by biological, behavioral, psychosocial, and environmental factors (23). Although there is still a need for longitudinal studies, recent meta-analyses have found that T1DM is associated with a high risk for dental caries $(24,25)$. The prevalence of dental caries among 538 children and adolescents with T1DM from 10 different studies worldwide was $67 \%$. The prevalence was the highest in South America (84\%) and the lowest in patients with diabetes having good metabolic control (47\%) (24). In another meta-analysis, T1DM patients had significantly higher levels of dental caries in permanent teeth but not in deciduous teeth than the non-diabetic group. However, no significant differences were found between patients with well-controlled and poorly controlled T1DM (25). Some studies have found correlations between metabolic control and diabetes course, together with dental caries stages (26-29). The divergent findings described above are probably related to the cut-offs of HbA1c as well as to age strata in the studies (25). The groups of T1DM children with HbA1c of $>10 \%$ exhibited more caries lesions and bleeding gums than the other groups (28).

Different species of bacteria, such as Streptococcus, Veillonella, Actinomyces, Granulicatella, Leptotrichia, Thiomonas, Bifidobacterium, and Prevotella, have been associated with the development of dental caries in children $(30,31)$. The majority of studies on T1DM patients were conducted using laboratory culture techniques or polymerase chain reaction (PCR) analysis (22, 32-35). Generally, patients with wellcontrolled diabetes have fewer decayed surfaces and lower counts of Streptococcus mutans, lactobacilli, and yeast than those with poorly controlled diabetes (33-35). Samples from the bottom of the oral cavity and dorsum of the tongue were collected from 50 T1DM children aged 10-18 years and assigned into two groups: well-controlled and poorly controlled groups. Twenty-five children were used as healthy controls. Collected samples were analyzed for total bacteria and different species of Streptococcus, 


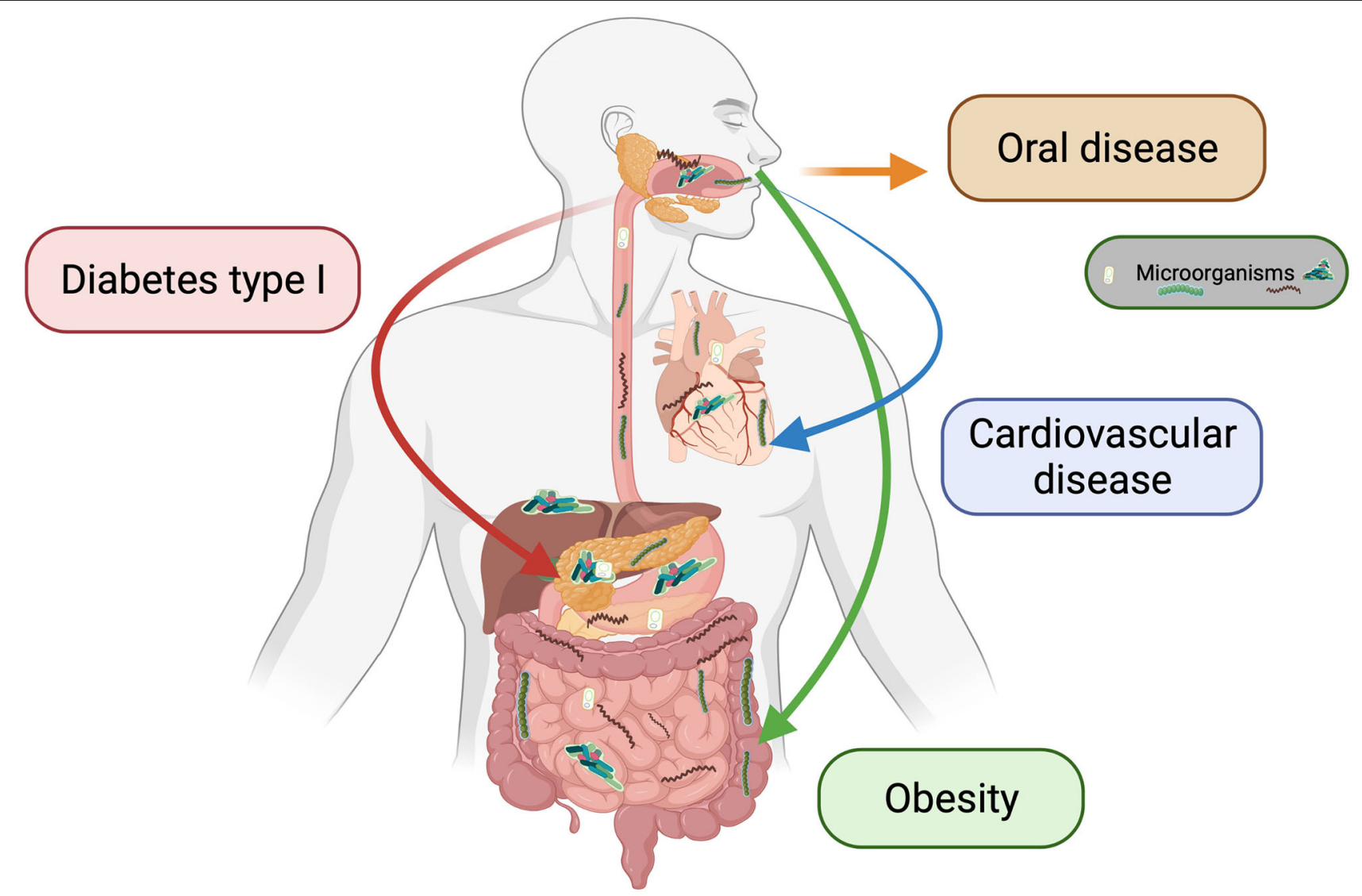

FIGURE 1 | Interplay between oral and systemic diseases. The oral cavity, particularly when caries and gingivitis/periodontitis are present, could act as microbial reservoir, interplaying with cardiovascular disease (via the bloodstream), obesity (via the digestive system), and diabetes type I (via the immune system).

Enterococcus, Staphylococcus, Candida, and anaerobic bacteria. The authors found an increased amount of Streptococcus mitis in T1DM children than in healthy children. A significantly higher number of different strains was isolated from diabetic groups, mainly in poorly controlled diabetes (22). Another study revealed significantly higher levels of dental plaque and higher counts of $S$. mutans in T1DM children with poor glycemic control than in the healthy control group. Candida albicans levels were not statistically different among the groups, but those with poor glycemic control showed an increased frequency of detection (32).

Some risk factors inherent to patients with diabetes could potentialize the development or progression of tooth decay. Of interest, diabetic children consume daily meals more frequently, which favors in the saliva: higher episodes of low $\mathrm{pH}$, lower concentration of bicarbonate, reduced unstimulated and stimulated secretion flow rates leading to xerostomia, increased glucose levels, lower levels of antimicrobial proteins such as lactoferrin and lysozyme, and bacterial proliferation (36-38).

Given the importance of the disturbances mentioned above, further scientific evidence is necessary to elucidate the relationship between the development of dental caries lesions in children with diabetes considering the associated factors.

\section{Plaque-Induced Gingivitis and T1DM}

Commonly, there is a symbiotic relationship between the host and the oral microbiome to maintain homeostasis, and a dysbiosis between the dental biofilm and the host's immuneinflammatory response may initiate gingivitis (39). In addition, poor nutrition can cause increased inflammation (40), and biofilm can accumulate rapidly in inflamed gingiva. The clinical signs (redness and edema) and symptoms of inflammation confined to the gingiva is reversible when the biofilm is disrupted or removed $(39,41)$. However, if gingivitis is not controlled, it can progress to periodontal disease comprising the periodontal ligament, cementum, and alveolar bone in older ages.

The primary parameter to evaluate the presence of gingivitis is bleeding on probing (BOP) $(41,42)$. A patient with an intact periodontium is diagnosed with gingivitis when the BOP score is $\geq 10 \%$. Localized gingivitis involves a BOP score of $10-30 \%$, whereas a score of $>30 \%$ is classified as generalized gingivitis (41). When only a few sites are affected by mild inflammation, the condition is referred to as incipient gingivitis $(39,41)$.

In adolescents, other local factors, such as dental caries, mouth breathing, crowding of the teeth, and tooth eruption can modify the incidence and severity of gingivitis. Significant changes in 
steroid hormone levels during puberty also have a transient effect on inflammation of the gingiva $(39,43)$.

Clinical studies have demonstrated that the presence of diabetes may be considered a risk factor for periodontal disease in children and adolescents $(44,45)$. Gingivitis is the predominant form of periodontal disease in childhood, and the level of glycemic control may be more important in determining the severity of gingival inflammation than the quality of plaque control (46-48).

Hyperglycemia causes a hyperinflammatory response in the presence of bacterial biofilm. Individuals with diabetes have impaired neutrophil and macrophage function, altered collagen production, exaggerated collagenase activity, hyperinflammatory responsive monocytes, and an increased release of proinflammatory cytokines $(49,50)$. Another factor that may modify host responses is the accumulation of advanced glycation endproducts (AGE) and their interaction with AGE receptors in children with diabetes (49).

Previous studies have shown that gram-positive species (e.g., Streptococcus spp., Actinomyces viscosus, Peptostreptococcus micros) and gram-negative species (e.g., Campylobacter gracilis, Fusobacterium nucleatum, Prevotella intermedia, Veillonella spp.) are associated with gingivitis (51). A clinical study showed that Capnocytophaga sputigena and Capnocytophaga ochracea were associated with gingivitis in children with T1DM and that glycemic and lipid parameters were higher in patients with T1DM, albeit within normal values (46).

Periodontopathogenic bacteria can cause direct damage to periodontal tissues or indirect tissue damage by inducing the release of inflammatory cytokines and other mediator bacteria (51). The transition from health to disease follows the principles of primary ecological succession, rather than the acquisition of new organisms (41), suggesting that clusters of bacteria may be a more robust discriminant of disease (41).

Of interest, poorly controlled diabetes may cause xerostomia due to hyposalivation. Xerostomia is indirectly related to gingival disease activity through the accumulation of dental plaque in young adults (52).

Despite an increase in the number of studies that have assessed the association between diabetes and gingival inflammation, no consensus has yet emerged about a possible causal relationship $(53,54)$. A recent systematic review and meta-analysis concluded that the severity of periodontal inflammation is higher in children and adolescents with T1DM than in healthy individuals. However, the authors did not provide strong evidence that periodontitis is a significant risk factor for T1DM in children (53). Other studies on childhood diabetes have also shown that gingival inflammation is higher in children with T1DM than in non-diabetic children $(44,45)$ and suggested that periodontal destruction can begin early in children with diabetes.

Regarding the influence of glycemic control elements on the presence of gingivitis, data are not conclusive, suggesting other factors, such as those related to patients' immunological responses (55). A recent study showed no significant differences in periodontal status between controlled and poorly controlled diabetic patients and healthy children (56). In a case-control study involving 80 children and adolescents (aged 5-18 years) with T1DM, a significant effect of diabetes on an increased risk of oral and periodontal diseases in children was not confirmed (57). In the same context, a comparative cross-sectional study on children with T1DM and non-diabetic children with mixed dentition, both sexes (7-13 years) and without a distinction of race demonstrated that the periodontal conditions were similar among patients in both groups, without statistical differences in any periodontal indexes (46). This study also demonstrated through microbiological analysis that red-complex bacteria were present at a few sites. Fusobacterium nucleatum and Campylobacter rectus were more frequently detected, and interleukin (IL)-6 levels were similar between the groups (46). On the other hand, an up-to-date research by Jensen et al. (58) demonstrated that worsening glycemic control is associated with increased severity of early markers of periodontal disease in children and adolescents with T1DM. In that study, it was also observed that glycemic control was related to the complexity and richness of the microbiota of the gingival plaque and lower brushing frequency, independent of glycated hemoglobin (HbAlc) (58). Thus, well-designed clinical studies are still required to clarify the interplay between diabetes and inflammation of gingival and periodontal tissues.

\section{EARLY CHILDHOOD CARIES AND OBESITY}

As mentioned above, dental caries is a major oral health problem and in the early childhood, is characterized by the presence of one or more deciduous teeth with the presence of a carious lesion, cavitated or not, in children under the age of 6 year (59).

It is important to highlight that primary teeth maintain the space for adequate development of the permanent dentition and are essential for the child's well-being, phonetics, esthetics, and mastication. Unfortunately, most early childhood caries (ECC) lesions remain untreated (59), leading to chronic pain, infections, and other comorbidities (60).

In the last 45 years, worldwide, obesity has increased threefold, and $\sim 38$ million children under the age of 5 years were overweight or obese in 2019 (61). While overweight is characterized by a body mass index (BMI) of the 97-99.9th percentile, obesity is defined by a BMI of $>99.9$ th percentile in those aged younger than 5 years (62). Overweight or obesity in childhood is considered a risk factor for adulthood obesity and might be directly related to diabetes and cardiovascular disorders.

The effect of obesity on functional and metabolic changes in the human body is an important topic to explore. As believed before, the adipose tissue is not only responsible for energy storage, but an endocrine organ, producing adipokines (leptin, adiponectin, visfatin, resistin, apelin). As weight gain is connected to increased adipose tissue mass, these hormones might probably be produced in higher concentrations, significantly affecting the metabolism of macronutrients (63) and causing a "metainflammation" (64). An usual consequence of obesity is the metabolic syndrome characterized by a clustering of risk factors (insulin resistance, hyperleptinemia, hypoadiponectinemia) predisposing individuals to the development of future comorbidities (64). 
A recent systematic review showed that children with high BMI scores had about a two-times higher chance of experiencing ECC than lean children (65). Despite both diseases (ECC and obesity) being complex and sharing a common risk factor (diet), microbial dysbiosis also plays a critical role (9), profoundly affecting disease course/development. Remarkably, the human oral and gut microbiomes present enormous complexity and several functions such as the development of immunity and defense against pathogens. Gut microorganisms also produce short chains of fatty acids that are important for energy metabolism, synthesis of vitamins, and fat storage (66). Unlike the human genome, which is relatively constant, the microbiome is dynamic and is altered by changes in development, environmental factors such as diet and use of antibiotics, and the response to disease (67).

Harboring billions of microbes (68), the oral cavity microbiome is composed mainly of the following phyla: Proteobacteria, Bacteroidetes, Firmicutes, Actinobacteria, and Fusobacteria $(69,70)$. Despite being a polymicrobial disease, the predominance of acidogenic and aciduric bacteria $(4,5,71)$ favors the demineralization process of the dental tissues after carbohydrate fermentation, leading to white chalky spot lesions that further progress into dentin cavitation $(4,5)$. Notably, it was estimated that children with severe ECC exhibited 94.5 phylotypes vs. 113.4 in caries-free children, suggesting that microbial variety and complexity in dental biofilm are significantly higher in healthy subjects (72). This is because carious lesions could act as retentive niches for cariogenic bacteria, which dominate as the disease progresses, leading to a decrease in the overall richness of the biofilm community (72).

While high numbers of mutans streptococci are significantly associated with early caries lesions, lactobacilli are linked to an advanced staged cavitation $(73,74)$. Conversely, quantitative PCR analysis of biofilm bacteria according to different stages of ECC indicated that $S$. mutans were also present in higher numbers in dentine caries lesion/cavitations, as well as Bifidobacterium spp. (75). Scardovia wiggsiae, a specie belonging to the phylum Actinobacteria, has also been linked to ECC (30), as well Veillonella, Prevotella, Porphyromonas, Actinomyces species, and the fungus $C$. albicans (76-79). Even with genetic sequencing using the $16 \mathrm{~S}$ ribosomal RNA gene, and better understood of the richness and diversity of the oral microbiome, S. mutans has still been identified as the most discriminatory specie between health and disease (80).

The classical main pathogens of dental caries, S. mutans and lactobacilli, belong to the Firmicutes phylum, which was found to be enhanced in samples collected from cavitated carious lesions (70). Interestingly, an increase in the abundance of the Firmicutes phylum, one of the largest in the gut microbiome, is commonly observed in childhood obesity $(8,10,81)$. In this respect, bacteria belonging to this phylum may be related to weight gain, such as an increase in the species of Eubacterium hallii, Clostridium leptum, and certain Lactobacillus species. Clostridium leptum is an important carbohydrate-fermenting bacterium belonging to the Clostridial IV set. Along with other intestinal microorganisms, they are capable of fermenting fiber and unabsorbed sugars from the diet, producing short-chain fatty acids that can act as an energy source for the human host, and can also influence intestinal epithelial function $(9,82)$. In line with this information, germ-free mice receiving a microbiota transplant increase their caloric uptake, energy harvest and body fat (83).

The microbiota could be considered an endocrine organ related to the maintenance of energy homeostasis and host immunity (84). It is understood that gut microorganisms are capable of 1 . increasing energy production from food, 2. contributing to subclinical inflammation, and 3. regulating fatty acid tissue composition $(85,86)$. Moreover, under dysbiotic conditions, the functioning of the intestinal barrier and gutassociated lymphoid tissues is altered, favoring the passage of lipopolysaccharides, which activate inflammatory pathways that might contribute to the development of insulin resistance (84). Additionally, the production of gastrointestinal peptides associated with satiety is also changed, leading to increased food intake.

It is important to highlight that the oral cavity and gut provide ideal niches for the largest microbiomes in the human body, due to the moist, warm, and nutrient-rich environments. The difference between them relies on the shedding characteristics of the mucosa vs. the non-shedding characteristics of the teeth. However, due to the arsenal of adhesive molecules, streptococci can colonize many types of surfaces (87). Intriguingly, some groups of bacteria could overlap in oral and stool samples (88-90), due to oral bacteria often being swallowed together with saliva and food during the digestion process. A recent study involving preschoolers investigated whether Firmicutes and Bacteroidetes levels in the mouth reflected the gut condition in obesity and ECC, demonstrating that Firmicutes phyla behave differently according to the nutritional status (obesity or eutrophy) and caries experience, and that dental biofilm and gut microbiome might share levels of similarity. In addition, the authors found significantly higher numbers of Firmicutes in obese children with ECC than in those with obesity and free of caries in both the mouth and gut (88).

The pivotal role of oral bacteria ectopically colonizing the gut remains unknown (91). In addition, it is challenging to distinguish between bacteria that truly reside in the gut and those that are temporarily present in the gut (92). In animal models, bacterial colonization success in the gut has been suggested to depend on their ability to metabolize dietary and host carbohydrates, as well as bile acids (93).

Although ECC and obesity are preventable, they continue to affect millions of children (59); therefore, studies involving common approaches should be conducted and will certainly be more effective. Moreover, the hypothesis that the mouth might act as a reservoir for intestinal pathogens that can aggravate diseases connected to the gut microflora (93) is of prime importance and should be further explored.

\section{CARIES AND CARDIOVASCULAR DISEASES}

The first common risk approach for cardiovascular pathologies and caries can be established by considering the individual's lifestyle, particularly eating habits. The high consumption of 
ultra-processed foods, fermentable carbohydrates, and saturated fats has led to an increase in the number of cases of hypertension, atherosclerosis, and cardiovascular diseases, as well as the number of individuals affected by caries (94).

Remarkably, bacteria found in cariogenic biofilms can synthesize extracellular polysaccharide matrix from dietary sugars, favoring the adhesion of multispecies microorganisms $(95,96)$. As described in the previous sections of the present review, when this biofilm is undisturbed, that is, when brushing and flossing are not frequent, the propensity for carious lesions is enhanced. In addition, when dental caries progresses until a severe stage the pulp, an organ full of nerves and capillaries, is exposed and there is higher risk of bacteremia via the bloodstream. This way, the typical pathogens associated with tooth decay, S. mutans and Lactobacillus spp., together with Veillonella spp., Scardovia spp. and other oral streptococci get access to other organs, such as the heart, causing an increase in the levels of systemic antibodies, and with possible development of a variety of cardiovascular disturbances, i.e., infectious endocarditis $(97,98)$.

Infectious endocarditis is characterized by endocardial surface contagions. Valves are the most affected structures, but other endocardial tissue locations might also be involved (99). Endocarditis is intimately linked to microorganisms in the group of oral streptococci, staphylococci, enterococci, gram-negative bacilli, some fungi (Candida spp.), fastidious microbes, and cultivable intracellular microorganisms such as Chlamydophila spp., S. mutans, and Staphylococcus aureus (99). Severe sepsis or septic shock has a mortality rate of $20-25 \%$ and is associated with microorganisms such as Staphylococcus aureus and nonhemolytic streptococci.

Curiously, S. mutans was the most frequently detected bacteria in atheromatous plaques and unhealthy heart valve tissues (100-102). When S. mutans and other oral bacteria enter the circulatory system (103) reaching the heart tissues, they easily adhere to heart valves, producing an insoluble dextran from blood glucose and forming biofilms (97). According to the composition and structure of the rhamnose glucose polysaccharide connected to the cell wall, $S$. mutans can be divided into four different serotypes: c, e, f, and k. Although serotype $c$ is the most common in the oral cavity, serotypes $\mathrm{e}$ and $\mathrm{f}$ are shown to invade primary human coronary artery endothelial cells. Intriguingly, invasive strains carry the surface protein with collagen- and laminin-binding activity (cnm) gene, which can bind to collagen and laminin in vitro, favoring adherence to endothelial tissues and triggering inflammatory responses, similar to other surface structures of S. mutans (104-106).

Another important oral disease that begins with the imbalance of the healthy microbiota in the subgingival environment is periodontitis. As already mentioned, it is an oral infectious disease that can develop in late childhood or adolescence, caused mainly by gram-negative bacteria, with the destruction of the tissues supporting the teeth as a result of an injury caused by the pathogenic biofilm. Hence, in the presence of periodontal disease, the junctional epithelium and connective tissue are not firm and the risk of bleeding is enhanced, favoring the access of oral microorganisms, especially $S$. mutans, to the capillaries and bloodstream $(97,103)$.

Chronic periodontitis can alter the lipid profile, contributing to the progression of atherosclerosis (107). Furthermore, the host's response to gram-negative periodontopathogens bacterial lipopolysaccharides is a pro-inflammatory response, with the production of IL-6, prostaglandin E2, and matrix metalloproteinases, culminating in tissue destruction. In addition, the production of IL-1 beta, IL-6, and tumor necrosis factor-alpha can promote hyperlipidemia, potentializing the risk of atherosclerosis, which is the main cause of heart disease. Studies have shown that cardiovascular problems, such as coronary heart disease, stroke, peripheral vascular disease, cardiomyopathy, atherosclerosis, and myocardial infarction, are linked to chronic infection and inflammation, which is the case in periodontitis $(95,108)$.

Antibiotic therapy is used for the treatment of diseases such as infectious endocarditis and sepsis. In this regard, we have to be mindful that due to the high resistance rate of some microorganisms in the infectious processes, the combination of antimicrobials may be necessary, as well as prolonged drug treatment to avoid recurrence. More than $50 \%$ of patients require surgery in cases of heart failure, uncontrolled infection, and embolism prevention (99).

Finally, it is important to point out that the oral cavity is a reservoir for complex commensal microbiota, which is a dysbiotic condition that favors caries and periodontitis development. Jointly with the presence of microbes in the mouth, relevant risk factors like sugar-rich food and lack of proper tooth brushing or flossing are also closely associated with the installation and progression of oral diseases. Regarding a common risk approach, a balanced diet with low to moderate fermentable carbohydrates intake/ultraprocessed foods not only reduces the chances of cariogenic biofilm formation, but contributes to improving the general functioning of the body. Thus, healthy gums and teeth are associated with a low risk of developing infectious oral diseases, bacteremia, and associated cardiovascular disturbances $(95,108)$.

\section{FINAL CONSIDERATIONS}

Altogether, in a critical point of view all the diseases described above are of high complexity and reinforce the holistic concept that the mouth could not be separated of the body. It should be prohibitive focusing too narrowly on single chronic diseases alone. In this regard, a multidisciplinary approach should be emphasized, bringing together healthcare professionals from different fields, with different expertise, such as dentists, physicians, nutritionists, psychologists and nurses. The organization and interrelationship between these professionals, will favor since the early diagnosis and effective preventive strategies, until assertive diagnosis and treatment plan, improving prognosis and patient's quality of life. 
It should be kept in mind that multidisciplinary teams have higher chances of meeting the demands of patients with complex care needs, attaining in the development of a special routine supporting their care goals. When the right attention in the communities is delivered, well-being is favored and unnecessary complicated treatments or hospitalizations could be avoided, reducing the oral/systemic health budget expenditure.

Of interest, the clinical practice based on scientific evidences requires the ability to locate information and appraise it critically. Literature reviews play important roles in this regard.

In summary, considering the relationship between the oral microbiome and chronic diseases driven by metabolic dysfunction in childhood, it should be highlighted that:

- Microbe establishment is linked to biological, behavioral, and psychosocial factors associated with an individual's environment.

\section{REFERENCES}

1. Deo PN, Deshmukh R. Oral microbiome: unveiling the fundamentals. J Oral Maxillofac Pathol. (2019) 23:122-8. doi: 10.4103/jomfp.JOMFP_152_19

2. Dubey S, Dubey S, Gupta A, Sharma V. Biofilm-Mediated dental diseases. In: Kumar S, Chandra N, Singh L, Hashmi M, Varma ASW, editors. Biofilms in Human Diseases: Treatment and Control (Cham: Springer). doi: 10.1007/978-3-030-30757-8_7

3. World Health Organization. Oral Health. (2020). Available online at: https:// www.who.int/news-room/fact-sheets/detail/oral-health (accessed May 28, 2021)

4. Selwitz RH, Ismail AI, Pitts NB. Dental caries. Lancet. (2007) 369:519. doi: 10.1016/S0140-6736(07)60031-2

5. Seow WK. Early childhood caries. Pediatr Clin North Am. (2018) 65:94154. doi: 10.1016/j.pcl.2018.05.004

6. Trombelli L, Farina R. A review of factors influencing the incidence and severity of plaque-induced gingivitis. Minerva Stomatol. (2013) 62:207-34.

7. Olsen I, Yamazaki K. Can oral bacteria affect the microbiome of the gut? J Oral Microbiol. (2019) 11:1586422. doi: 10.1080/20002297.2019.1586422

8. Gallardo-Becerra L, Cornejo-Granados F, García-López R, Valdez-Lara A, Bikel S, Canizales-Quinteros S, et al. Metatranscriptomic analysis to define the secrebiome, and 16S rRNA profiling of the gut microbiome in obesity and metabolic syndrome of Mexican children. Microb Cell Fact. (2020) 19:61. doi: 10.1186/s12934-020-01319-y

9. Indiani CMDSP, Rizzardi KF, Castelo PM, Ferraz LFC, Darrieux M, et al. Childhood obesity and firmicutes/bacteroidetes ratio in the gut microbiota: a systematic review. Child Obes. (2018) 14:501-9. doi: 10.1089/chi.2018.0040

10. Riva A, Borgo F, Lassandro C, Verduci E, Morace G, Borgui E, et al. Pediatric obesity is associated with an altered gut microbiota and discordant shifts in firmicutes populations. Environ Microbiol. (2017) 19:95105. doi: 10.1111/1462-2920.13463

11. Ignacio A, Fernandes MR, Rodrigues VA, Groppo FC, Cardoso AL, Avila-Campos MJ, et al. Correlation between body mass index and faecal microbiota from children. Clin Microbiol Infect. (2016) 22:e18. doi: 10.1016/j.cmi.2015.10.031

12. Vaarala O. Gut microbiota and type 1 diabetes. Rev Diabet StudWinter. (2012) 9:251-9. doi: 10.1900/RDS.2012.9.251

13. Pietiäinen M, Liljestrand JM, Kopra E, Pussinen PJ. Mediators between oral dysbiosis and cardiovascular diseases. Eur J Oral Sci. (2018) 1:2636. doi: $10.1111 /$ eos. 12423

14. American Diabetes Association. Classification and diagnosis of diabetes: standards of medical care in diabetes. Diabetes Care. (2020) 43:S1431. doi: $10.2337 / \mathrm{dc} 20-\mathrm{S} 002$
- A better understanding of the human microbiome could indicate the potential microorganisms connected to health or disease.

- Current molecular biology technologies favor knowledge acquisition concerning microbial diversity and its relationship with physiopathological conditions, but the exact mechanism connecting oral diseases and microbiota to chronic diseases driven by metabolic dysfunction during childhood is far from being completely understood.

\section{AUTHOR CONTRIBUTIONS}

$\mathrm{CD}$ and AR: conceptualization. FS, TP, SB, LT, JH, and CD: writing-original draft preparation. FS, TP, and $\mathrm{CD}$ : writing-review and editing. $\mathrm{TP}$ and $\mathrm{CD}$ : supervision. All authors significantly contributed to the manuscript preparation and approved the final version.

15. Campbell-Thompson M, Rodriguez-Calvo T, Battaglia M. Abnormalities of the exocrine pancreas in type 1 diabetes. Curr Diab Rep. (2015) 15:79. doi: 10.1007/s11892-015-0653-y

16. Oikarinen M, Tauriainen S, Oikarinen S, Honkanen T, Collin P, Rantala I, et al. Type 1 diabetes is associated with enterovirus infection in gut mucosa. Diabetes. (2012) 61:687-91. doi: 10.2337/db11-1157

17. Mattila M, Niinistö S, Takkinen HM, Tapanainen H, Reinivuo H, Åkerlund $\mathrm{M}$, et al. Maternal nitrate and nitrite intakes during pregnancy and risk of islet autoimmunity and type 1 diabetes: the DIPP cohort study. J Nutr. (2020) 150:2969-76. doi: 10.1093/jn/nxaa250

18. Rabbone I, Traversi D, Scaioli G, Vallini C, Carletto G, Masante I, et al. Microbiota, epidemiological and nutritional factors related to ketoacidosis at the onset of type 1 diabetes. Acta Diabetol. (2020) 57:133749. doi: 10.1007/s00592-020-01555-z

19. World Health Organization. Global Report on Diabetes, Part 1 Global Burden of Diabetes. (2016). Available online at: https://www.who.int/publications/i/ item/9789241565257 (accessed May, 28, 2021).

20. Kahanovitz L, Sluss PM, Russell SJ. Type 1 diabetes - a clinical perspective. Point Care. (2017) 16:37-40. doi: 10.1097/POC.0000000000000125

21. Kilian M, Chapple IL, Hannig M, Marsh PD, Meuric V, Pedersen AM, et al. The oral microbiome - an update for oral healthcare professionals. Br Dent J. (2016) 221:657-66. doi: 10.1038/sj.bdj.2016.865

22. Pachoński M, Koczor-Rozmus A, Mocny-Pachońska K, Łanowy P, Mertas A, Jarosz-Chobot P. Oral microbiota in children with type 1 diabetes mellitus. Pediatr Endocrinol Diabetes Metab. (2021) 21:43494. doi: 10.5114/pedm.2021.104343

23. Machiulskiene V, Campus G, Carvalho JC, Dige I, Ekstrand KR, Jablonski-Momeni A, et al. Terminology of dental caries and dental caries management: consensus report of a workshop organized by orca and cariology research group of IADR, Caries Res. (2020) 54:714. doi: 10.1159/000503309

24. Wang Y, Xing L, Yu H, Zhao L. Prevalence of dental caries in children and adolescents with type 1 diabetes: a systematic review and meta-analysis. BMC Oral Health. (2019) 19:213. doi: 10.1186/s12903-019-0903-5

25. Coelho AS, Amaro IF, Caramelo F, Paula A, Marto CM, Ferreira $\mathrm{MM}$, et al. Dental caries, diabetes mellitus, metabolic control and diabetes duration: a systematic review and meta-analysis. J Esthet Restor Dent. (2021) 32:291-390. doi: 10.1111/jerd. 12562

26. Malvania EA, Sheth SA, Sharma AS, Mansuri S, Shaikh F, Sahani S. Dental caries prevalence among type II diabetic and nondiabetic adults attending a hospital. J Int Soc Prevent Commun Dent. (2016) 6:S2326. doi: 10.4103/2231-0762.197202 
27. Kogawa EM, Grisi DC, Falcao DP, Amorim IA, Rezende TMB, Rodrigues IC, et al. Impact of glycemic control on oral health status in type 2 diabetes individuals and its association with salivary and plasma levels of chromogranin a. Arch Oral Biol. (2016) 62:10-9. doi: 10.1016/j.archoralbio.2015.11.005

28. Carneiro VL, Fraiz FC, Ferreira FM, Pintarelli TP, Oliveira AC, Boguszewski MC. The influence of glycemic control on the oral health of children and adolescents with diabetes mellitus type 1. Arch Endocrinol Metab. (2015) 59:535-40. doi: 10.1590/2359-3997000000117

29. Twetman S, Petersson GH, Bratthall D. Caries risk assessment as a predictor of metabolic control in young type 1 diabetics. Diabet Med J Br Diabetic Assoc. (2005) 22:312-5. doi: 10.1111/j.1464-5491.2005.01419.x

30. Tanner ACR, Kent RL, Holgerson PL, Hughes CV, Chalmers NI, Johansson I. Microbiota of severe early childhood caries before and after therapy. J Dent Res. (2011) 90:1298-305. doi: 10.1177/0022034511421201

31. Yang F, Zeng X, Ning K, Liang-Li K, Chi-Lo C, Wang W, et al. Saliva microbiomes distinguish caries-active from healthy human populations. ISME J. (2011) 6:1-10. doi: 10.1038/ismej.2011.71

32. Babatzia A, Papaioannou W, Stavropoulou A, Pandis N, Kanaka-Gantenbein C, Papagiannoulis L, et al. Clinical and microbial oral health status in children and adolescents with type 1 diabetes mellitus. Int Dent J. (2020) 70:136-44. doi: 10.1111/idj.12530

33. Siudikiene J, Machiulskiene V, Nyvad B, Tenovuo J, Nedzelskiene I. Dental caries increments and related factors in children with type 1 diabetes mellitus. Caries Res. (2008) 42:354-62. doi: 10.1159/000151582

34. Siudikiene J, Machiulskiene V, Nyvad B, Tenovuo J, Nedzelskiene I. Dental caries and salivary status in children with type 1 diabetes mellitus, related to the metabolic control of the disease. Eur J Oral Sci. (2006) 114:814. doi: 10.1111/j.1600-0722.2006.00277.x

35. Bolgül BS, Celenk S, Ayna BE, Atakul F, Uysal E. Evaluation of caries risk factors and effects of a fluoride-releasing adhesive material in children with insulin-dependent diabetes mellitus (IDDM): initial first-year results. Acta Odontol Scand. (2004) 70:289-92. doi: 10.1080/00016350410001766

36. López ME, Colloca ME, Páez RG, Schallmach JN, Koss MA, Chervonagura A. Salivary characteristics of diabetic children. Braz Dent J. (2003) 14:2631. doi: 10.1590/S0103-64402003000100005

37. Moreira AR, Passos IA, Sampaio FC, Soares MS, Oliveira RJ. Flow rate, $\mathrm{pH}$ and calcium concentration of saliva of children and adolescents with type 1 diabetes mellitus. Braz J Med Biol Res. (2009) 42:70711. doi: $10.1590 / \mathrm{S} 0100-879 \mathrm{X} 2009005000006$

38. Zalewska A, Knaś M, Kuzmiuk A, Waszkiewicz N, Niczyporuk M, Waszkiel $\mathrm{D}$, et al. Salivary innate defense system in type 1 diabetes mellitus in children with mixed and permanent dentition. Acta Odontol Scand. (2013) 71:1493-500. doi: 10.3109/00016357.2013.773071

39. Murakami S, Mealey BL, Mariotti A, Chapple ILC. Dental plaque-induced gingival conditions. J Periodontol. (2018) 89:S17-S27. doi: 10.1002/JPER.17-0095

40. Loos BG, Van Dyke TE. The role of inflammation and genetics in periodontal disease. Periodontology. (2020) 83:26-39. doi: 10.1111/prd.12297

41. Trombelli L, Farina R, Silva CO, Tatakis DN. Plaque-induced gingivitis: case def- inition and diagnostic considerations. J Periodontol. (2018) 89:S4673. doi: 10.1002/JPER.17-0576

42. Caton J, Armitage G, Berglundh T, Chappkle ILC, Jepsen S, Kornman KS, et al. A new classification scheme for periodontal and peri-implant diseases and conditions - introduction and key changes from the 1999 classification. J Periodontol. (2018) 89:S1-8. doi: 10.1002/JPER.18-0157

43. Gusberti FA, Syed SA, Bacon G, Grossman N, Loesche WJ. Puberty gingivitis in insulin-dependent diabetic children. I. Cross-sectional observations. J Periodontol. (1983) 54:714-20. doi: 10.1902/jop.1983.54.12.714

44. Lalla E, Cheng B, Lal S, Kaplan S, Softness B, Greenberg E, et al. Diabetes mellitus promotes periodontal destruction in children. J Clin Periodontol. (2007) 34:294-8. doi: 10.1111/j.1600-051X.2007.01054.x

45. Lalla E, Cheng B, Lal S, Tucker S, Greenberg E, Goland R, et al. Periodontal changes in children and adolescents with diabetes: a case-control study. Diabetes Care. (2006) 29:295-9. doi: 10.2337/diacare.29.02.06.dc05-1355

46. Duque C, João MF, Camargo GA, Teixeira GS, Machado TS, Azevedo RS, et al. Microbiological, lipid and immunological profiles in children with gingivitis and type 1 diabetes mellitus. J Appl Oral Sci. (2017) 25:21726. doi: 10.1590/1678-77572016-0196

47. Ervasti T, Knuutila M, Pohjamo L, Haukipuro K. Relation between control of diabetes and gingival bleeding. J Periodontol. (1985) 56:1547. doi: $10.1902 /$ jop.1985.56.3.154

48. Cianciola LJ, Park BH, Bruck E, Mosovich L, Genco RJ. Prevalence of periodontal disease in insulin-dependent diabetes mellitus (juvenile diabetes). J Am Dent Assoc. (1982) 104:65360. doi: 10.14219/jada.archive.1982.0240

49. Albandar JM, Susin C, Hughes FJ. Manifestations of systemic diseases and conditions that affect the periodontal attachment apparatus: case definitions and diagnostic considerations. J Periodontol. (2018) 89:S183203. doi: 10.1002/JPER.16-0480

50. Taylor JJ, Preshaw PM, Lalla E. A review of the evidence for pathogenic mechanisms that may link periodontitis and diabetes. J Periodontol. (2013) 84:S113-34. doi: 10.1902/jop.2013.134005

51. Tatakis DN, Kumar PS. Etiology and pathogenesis of periodontal diseases. Dent Clin North Am. (2005) 49:491-516. doi: 10.1016/j.cden.2005. 03.001

52. Mizutani S, Ekuni D, Tomofuji T, Azuma T, Kataoka K, Yamane M, et al. Relationship between xerostomia and gingival condition in young adults. $J$ Periodontal Res. (2015) 50:74-9. doi: 10.1111/jre.12183

53. Rapone B, Corsalini M, Converti I, Loverro MT, Gnoni A, Trerotoli $\mathrm{P}$, et al. Does periodontal inflammation affect type 1 diabetes in childhood and adolescence? A meta-analysis. Front Endocrinol. (2020) 11:278. doi: 10.3389/fendo.2020.00278

54. Novotna M, Podzimek S, Broukal Z, Lencova E, Duskova J. Periodontal diseases and dental caries in children with type 1 diabetes mellitus. Mediators Inflamm. (2015) 2015:379626. doi: 10.1155/2015/379626

55. Dias Rosas CY, Cardenas VE, Cataneda-Delgado J, AguilieraGalaviz LA, Aceves MMC. Den Dental, periodontal and salivary conditions in diabetic children associated with metabolic control variables and nutritional plan adherence. Eur J Paediatr Dent. (2018) 19:119-26. doi: 10.23804/ejpd.2018.19.02.05

56. Pachoński M, Jarosz-Chobot P, Koczor-Rozmus A, Łanowy P, MocnyPachońska K. Dental caries and periodontal status in children with type 1 diabetes mellitus. Pediatr Endocrinol Diabetes Metab. (2020) 26:3944. doi: 10.5114/pedm.2020.93249

57. Rafatjou R, Ravazi Z, Tayebi S, Khalili M. Dental health status and hygiene in children and adolescents with type I diabetes mellitus. J Res Health Sci. (2016) 16:122-6.

58. Jensen ED, Selway CA, Allen G, Bednarz J, Weyrich LS, Gue S,Pena AS, et al. Early markers of periodontal disease and altered oral microbiota are associated with glycemic control in children with type 1 diabetes. Pediatr Diabetes. (2020) 22:474-81. doi: 10.1111/pedi.13170

59. Pitts N, Baez R, Diaz-Guallory, C, Donly KJ, Feldens A, et al. Early childhood caries: IAPD Bangkok declaration. Int J Paediatr. (2019) 29:3846. doi: 10.1111/ipd.12490

60. Nicolae A, Levin L, Wong PD, Dave MG, Taras J, Mistry C, et al. Identification of early childhood caries in primary care settings. Paediatr Child Health. (2018) 23:111-5. doi: 10.1093/pch/pxx155

61. World Health Organization. Obesity and Overweight. (2020). Available online at: https://www.who.int/news-room/fact-sheets/detail/obesity-andoverweight (accessed May 28, 2021).

62. World Health Organization and Multicentre Growth Reference Study Group. Who Child Growth Standards: Length/Height-for-Age, Weight-forAge, Weight-for-Length, Weight-for-Height and Body Mass Index-for-Age: Methods and Development. (2006). Available online at: https://www.who.int/ childgrowth/standards/Technical_report.pdf?ua=1 (accessed May 27, 2021).

63. Singla P, Bardoloi A, Parkash AA. Metabolic effects of obesity: a review. World J Diabetes. (2010) 1:76-88. doi: 10.4239/wjd.v1.i3.76

64. Uranga RM, Keller JN. The complex interactions between obesity, metabolism and the brain. Front Neurosci. (2019) 13:513. doi: $10.3389 /$ fnins.2019.00513

65. Manohar N, Hayen A, Fahey P, Arora A. Obesity and dental caries in early childhood: a systematic review and meta-analyses. Obesity. (2020) 21:e12960. doi: 10.1111/obr.12960 
66. Amon P, Sanderson I. What is the microbiome? Arch Dis Child Educ Pract Ed. (2017) 102:257-60. doi: 10.1136/archdischild-2016-311643

67. Gilbert JA, Blaser MJ, Caporaso JG, Jansson JK, Lynch SV, Knight R. Current understanding of the human microbiome. Nat Med. (2018) 24:4. doi: $10.1038 / \mathrm{nm} .4517$

68. Zhang Y, Wang X, Li H, Ni C, Du Z, Yan F. Human oral microbiota and its modulation for oral health. Biomed Pharmacother. (2018) 99:88393. doi: 10.1016/j.biopha.2018.01.146

69. Wang Y, Wang S, Wu C, Chen X, Duan Z, Xu Q, et al. Oral microbiome alterations associated with early childhood caries highlight the importance of carbohydrate metabolic activities. mSystems. (2019) 4:e0045019. doi: $10.1128 / \mathrm{mSystems.00450-19}$

70. Hurley E, Barrett MPJ, Kinirons M, Whelton H, Ryan CA, Stanton C, et al. Comparison of the salivary and dentinal microbiome of children with severeearly childhood caries to the salivary microbiome of caries-free children. BMC Oral Health. (2019) 19:13. doi: 10.1186/s12903-018-0693-1

71. Parisotto TM, Steiner-Oliveira C, Silva CM, Rodrigues LK, Nobre-dosSantos M. Early childhood caries and mutans streptococci: a systematic review. Oral Health Prev Dent. (2010) 8:59-70.

72. Li Y, Ge Y, Saxena D, Caufield PW. Genetic profiling of the oral microbiota associated with severe early-childhood caries. J Clin Microbiol. (2007) 45:817. doi: 10.1128/JCM.01622-06

73. Parisotto TM, Steiner-Oliveira C, Duque C, Peres RCR, Rodrigues LKA, Nobre-dos-Santos M. Relationship among microbiological composition and presence of dental plaque, sugar exposure, social factors and different stages of early childhood caries. Arch Oral Biol. (2010) 55:36573. doi: 10.1016/j.archoralbio.2010.03.005

74. Ledder RG, Kampoo K, Teanpaisan R, McBain AJ. Oral microbiota in severe early childhood caries in thai children and their families: a pilot study. Front Microbiol. (2018) 9:2420. doi: 10.3389/fmicb.2018.02420

75. Neves BG, Stipp RN, Bezerra DDS, Guedes SFF, Rodrigues LKA. Quantitative analysis of biofilm bacteria according to different stages of early childhood caries. Arch Oral Biol. (2018) 96:155-61. doi: 10.1016/j.archoralbio.2018.09.007

76. Ma C, Chen F, Zhang Y, Sun X, Tong P, Si Y, et al. Comparison of oral microbial profiles between children with severe early childhood caries and caries-free children using the human oral microbe identification microarray. PLoS ONE. (2015) 10:e0122075. doi: 10.1371/journal.pone.0122075

77. Agnello M, Marques J, Cen L, Mittermuller B, Huang A, Chaichanasakul TN, et al. Microbiome associated with severe caries in canadian first nations children. J Dent Res. (2017) 96:1378-85. doi: 10.1177/0022034517718819

78. Xiao J, Grier A, Faustoferri RC, Alzoubi S, Gill AL, Feng C, et al. Association between oral candida and bacteriome in children with severe ECC. J Dent Res. (2018) 97:1468-76. doi: 10.1177/0022034518790941

79. de Jesus VC, Shikder R, Oryniak D, Mann K, Alamri A, Mittermuller B, et al. Sex-Based diverse plaque microbiota in children with severe caries. J Dent Res. (2020) 99:703-12. doi: 10.1177/0022034520908595

80. Dashper SG, Mitchell HL, Lê Cao KA, Carpenter L, Gussy MG, Calache H, et al. Temporal development of the oral microbiome and prediction of early childhood caries. Sci Rep. (2019) 24:19732. doi: 10.1038/s41598-019-56233-0

81. da Silva CC, Monteil MA, Davis EM. Overweight and obesity in children are associated with an abundance of firmicutes and reduction of bifidobacterium in their gastrointestinal microbiota. Child Obes. (2020) 16:204-10. doi: 10.1089/chi.2019.0280

82. den Besten G, van Eunen K, Groen AK, Venema K, Reijngoud DJ, Bakker BM. The role of short-chain fatty acids in the interplay between diet, gut microbiota, and host energy metabolism. J Lipid Res. (2013) 54:2325-40. doi: 10.1194/jlr.R0 36012

83. Turnbaugh PJ, Ley RE, Mahowald MA, Magrini V, Mardis ER, Gordon JI. An obesity-associated gut microbiome with increased capacity for energy harvest. Nature. (2006) 444:1027-31. doi: 10.1038/nature05414

84. Gomes AC, Hoffmann C, Mota JF. The human gut microbiota: metabolism and perspective in obesity. Gut Microbes. (2018) 4:308-25. doi: 10.1080/19490976.2018.1465157

85. Cani PD, Osto M, Geurts L, Everard A. Involvement of gut microbiota in the development of low-grade inflammation and type 2 diabetes associated with obesity. Gut Microbes. (2012) 3:279-88. doi: 10.4161/gmic.19625
86. Castaner O, Goday A, Park YM, Lee SH, Magkos F, Shiow STE, et al. The gut microbiome profile in obesity: a systematic review. Int J Endocrinol. (2018) 2018:4095789. doi: 10.1155/2018/4095789

87. Abranches J, Zeng L, Kajfasz JK, Palmer SR, Chakraborty B, Wen ZT, et al. Biology of oral streptococci. Microbiol Spectr. (2018) 6:118. doi: 10.1128/microbiolspec.GPP3-0042-2018

88. Rizzardi KF, Indiani CMSP, Mattos-Graner RO, de Sousa ET, Nobre-dosSantos M, et al. Firmicutes levels in the mouth reflect the gut condition with respect to obesity and early childhood caries. Front Cell Infect Microbiol. (2021) 11:593734. doi: 10.3389/fcimb.2021.593734

89. Indiani C, Rizzardi KF, Crescente CL, Steiner-Oliveira C, Nobre-Dos-Santos M, Parisotto TM. Relationship between mutans streptococci and lactobacilli in the oral cavity and intestine of obese and eutrophic children with early childhood caries-preliminary findings of a cross-sectional study. Front Pediatr. (2020) 8:588965. doi: 10.3389/fped.2020.588965

90. Segata N, Haake SK, Mannon P, Lemon KP, Waldron L, Gevers D, et al. Composition of the adult digestive tract bacterial microbiome based on seven mouth surfaces, tonsils, throat and stool samples. Genome Biol. (2012) 13:R42. doi: 10.1186/gb-2012-13-6-r42

91. Atarashi K, Suda W, Luo C, Kawaguchi T, Motoo I, Narushima $\mathrm{S}$, et al. Ectopic colonization of oral bacteria in the intestine drives TH1 cell induction and inflammation. Science. (2017) 358:359-65. doi: 10.1126/science.aan4526

92. Prodan A, Levin E, Nieuwdorp M. Does disease start in the mouth, the gut or both? Elife. (2019) 8:e45931. doi: 10.7554/eLife.45931

93. Seedorf H, Griffin NW, Ridaura VK, Reyes A, Cheng J, Rey FE, et al. Bacteria from diverse habitats colonize and compete in the mouse gut. Cell. (2014) 159:253-66. doi: 10.1016/j.cell.2014.09.008

94. Ravaghi V, Rezaee A, Pallan M, Morris AJ. Childhood obesity and dental caries: na ecological investigation of the shape and moderators of the association. BMC Oral Health. (2020) 20:338. doi: 10.1186/s12903-020-01329-7

95. Raidac A, Kapila Y. The orallome and its dysbiosis: new insights into oral microbiome-host interactions. Comput Struct Biotechnol J. (2021) 19:133560. doi: 10.1016/j.csbj.2021.02.010

96. Lemos JA, Palmer SR, Zeng L, Wen ZT, Kajfasz JK, Freires IA, et al. The biology of Streptococcus mutans. Microbiol Spectr. (2019) 7:126. doi: 10.1128/microbiolspec.GPP3-0051-2018

97. Folwaczny M, Bauer F, Grünberg C. Significance of oral health in adult patientes with congenital heart disease. Cardiovac Diagn Ther. (2019) 9:S377-87. doi: 10.21037/cdt.2018.09.17

98. Chen X, Daliri EBM, Kim N, Kim JR, Yoo D, Oh DH. Microbial etiology and prevention of dental caries: exploiting natural products to inhibit cariogenic biofilms. Pathogens. (2020) 9:569. doi: 10.3390/pathogens9070569

99. Cuervo G, Escrihuela-Vidal F, Gudiol C, Carrataià J. Current challenges in the management of infective endocarditis. Front Med. (2021) 8:641243. doi: 10.3389/fmed.2021.641243

100. Nakano $K$, Inaba $H$, Nomura $R$, Nemoto $H$, Takeda $M$, Yoshioka $H$, et al. Detection of cariogenic Streptococcus mutans in extirpated heart valve and atheromatous plaque specimens. J Clin Micro Biol. (2006) 44:33137. doi: 10.1128/JCM.00377-06

101. Nemoto H, Nakano K, Nomura R, Ooshima T. Molecular characterization of Streptococcus mutans strains isolated from the heart valve of an infective endocarditis patient. J Med Microbiol. (2008) 57:891-5. doi: 10.1099/jmm.0.47836-0

102. Nakano K, Ooshima T. Serotype classification of Streptococcus mutans and its detection outside the oral cavity. Future Microbiol. (2009) 4:891902. doi: $10.2217 / \mathrm{fmb} .09 .64$

103. Nomura R, Matayoshi S, Otsugu M, Kitamura T, Noboru T, Nakano K. Contribuition of severe dental caries induced by Streptococcus mutans to the pathogenicity of infective endocarditis. Infect Immun. (2020) 88:e0089719. doi: 10.1128/IAI.00897-19

104. Engels-Deutsch M, Pini A, Yamashita Y, Shibata Y, Haikel Y, SchollerGuinard $M$, et al. Insertional inactivation of pac and $\mathrm{rmlB}$ genes reduces the release of tumor necrosis factor alpha, interleukin-6, and interleukin-8 induced by Streptococcus mutans in monocytic, dental pulp, and periodontal ligament cells. Infect Immun. (2003) 71:516977. doi: 10.1128/IAI.71.9.5169-5177.2003 
105. Shun CT, Lu SY, Yeh CY, Chiang CP, Chia JS, Chen JY. Glucosyltransferases of viridans streptococci are modulins of interleukin-6 induction in infective endocarditis. Infect. Immun. (2005) 73:3261-70. doi: 10.1128/IAI.73.6.3261-327 0.2005

106. Abranches J, Miller JH, Martinez AR, Simpson-Haidaris PJ, Burne RA, Lemos JA. The collagen-binding protein Cnm is required for Streptococcus mutans adherence to and intracellular invasion of human coronary artery endothelial cells. Infect Immun. (2011) 79:2277-84. doi: 10.1128/IAI.00 $767-10$

107. Dörfer C, Benz C, Aida J, Campard G. The relationship of oral health with general health and NCDs: a brief review. Int Dent J. (2017) 2:14-8. doi: 10.1111/idj. 12360

108. Haque M, Sartelli M, Haque SZ. Dental infection and resistanceglobal health consequences. Dent J. (2019) 7:22. doi: 10.3390/dj70 10022
Conflict of Interest: The authors declare that the research was conducted in the absence of any commercial or financial relationships that could be construed as a potential conflict of interest.

Publisher's Note: All claims expressed in this article are solely those of the authors and do not necessarily represent those of their affiliated organizations, or those of the publisher, the editors and the reviewers. Any product that may be evaluated in this article, or claim that may be made by its manufacturer, is not guaranteed or endorsed by the publisher.

Copyright (@ 2021 Sabella, de Feiria, Ribeiro, Theodoro, Höfling, Parisotto and Duque. This is an open-access article distributed under the terms of the Creative Commons Attribution License (CC BY). The use, distribution or reproduction in other forums is permitted, provided the original author(s) and the copyright owner(s) are credited and that the original publication in this journal is cited, in accordance with accepted academic practice. No use, distribution or reproduction is permitted which does not comply with these terms. 